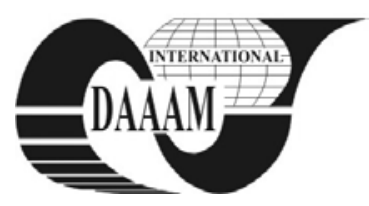

\title{
COMPARISON OF SER (SYMBOL ERROR RATE) IN SC-FDE AND OFDMA MODULATION SCHEMES
}

\author{
BAHTOVSKI, A[leksandr]
}

\begin{abstract}
In this article, I investigated the LTE uplink and performed link level simulations of Single Carrier Frequency Domain Equalization (SC-FDE) and Single Carrier Frequency Division Multiple Access (SC-FDMA) in comparison with Orthogonal Frequency Division Multiplexing (OFDM). The comparison has been done in terms of Signal-to-Noise Ratio (SNR) and Symbol Error Rate (SER).

Key words: equalization, telecommunication, wireless technology, symbol error rate simulation, SC-FDE, OFDMA, modulation schemes
\end{abstract}

\section{INTRODUCTION}

We are at the dawn of a new decade that will bring to mass market the mobile broadband innovations introduced over the last several years. 3G technology has shown its power and potential of always-on, everyplace network connectivity and has ignited a massive wave of industry innovation that spans devices, applications, Internet integration, and new business models.

Mobile communication technology has evolved rapidly in the last few years with an ever-increasing number of ways for users to access Internet services at any place and any time on mobile phones with an improved quality of offered services. In order to fulfill this, wireless telecommunication industry worked hard and defined a new air interface for mobile communications which enhances the overall system performance by increasing the capacity of the system along with improving spectral efficiencies while reducing latencies.

\section{WIRELINE VS. WIRELESS TECHNOLOGY}

Wireless technology plays a main role in networking and communications even though the existence of wireline technology which includes optical fibers with their advantages. The overwhelming global success of mobile telephony and the growing adoption of mobile data conclusively demonstrates the desire for mobile-oriented communications. Mobile broadband combines the new necessity of high-speed data services with mobility. Thus, providing limitless opportunities when considering the many diverse markets mobile broadband can successfully address.

Compared to wireless networks, wireline networks have always had greater capacity and faster data delivery.

The Long Term Evolution (LTE) is an evolution of the third generation technology based on Wideband Code Division Multiple Access (WCDMA). LTE uses OFDM for downlink, i.e. from base station to the terminal (Tanvir, 2007). There are three physical channels: Physical Downlink Shared Channel (PDSCH), Physical Multicast Channel (PMCH), Physical Broadcast Channel (PBCH) in the downlink used for data transmission, broadcast transmission and system information within a cell. The modulation schemes used are Quadrature Phase Shift Keying (QPSK), 16-Quadrature Amplitude Modulation (16-QAM) and 64-QAM.

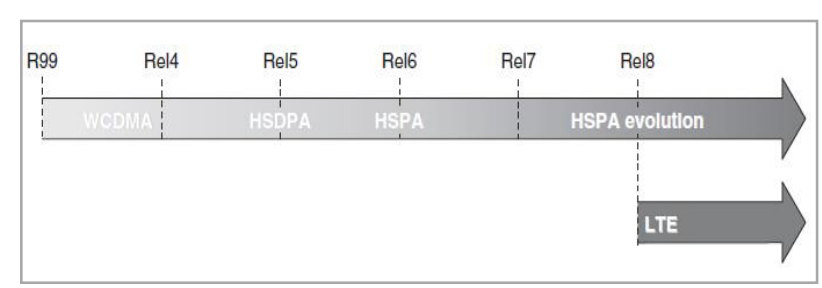

Fig. 1. LTE and evolution of HSPA

LTE uses a precoded version of Orthogonal Frequency Division Multiplexing (OFDM) using a single carrier for uplink called Single Carrier Frequency Division Multiplexing (SCFDMA). SC-FDMA is used to minimize Peak-to-Average Power Ratio (PAPR) caused by OFDM. PAPR is the ratio of peak signal power to the average signal power. There are two physical channels, Physical Random Access Channel (PRACH) and Physical Uplink Synchronization Channel (PUSCH), used in the LTE uplink. For initial access PRACH is used whereas when the User Equipment (UE) is not synchronized the data is send on PUSCH. The modulations schemes used for LTE uplink are QPSK, 16-QAM, 64-QAM.

\section{EVOLUTION PATH OF MOBILE TECHNOLOGIES}

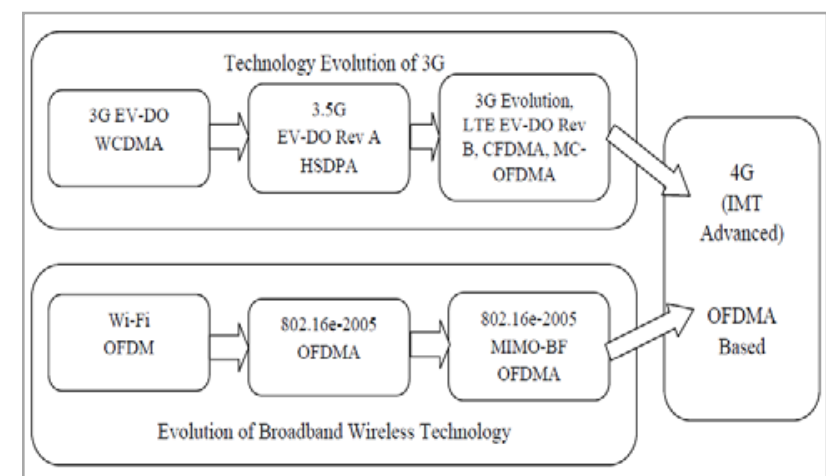

Fig. 2. Evolution Path of Mobile Technologies towards 4G

\section{COMPARISON OF SER (SIMBOL ERROR RATE) - SIMULATION}

SC-FDE is frequency domain equalization technique used for reduction of frequency selective fading effects in LTE uplink. SC-FDE has similar spectral efficiency and performances as OFDM (Bahtovski, 2011). However, SC-FDE has certain advantages upon OFDR due to usage of DFT and IDTF in the receiver. Block diagrams used in SC-FDE and OFDM simulation are shown in Fig.3 and Fig.4. Block similarity can be noted on the figures; both techniques are using same signal processing blocks. The parameters are chosen only for $5 \mathrm{MHz}$ transmission bandwidth of LTE system. The number of iterations used in the simulation are $10^{4}$. 


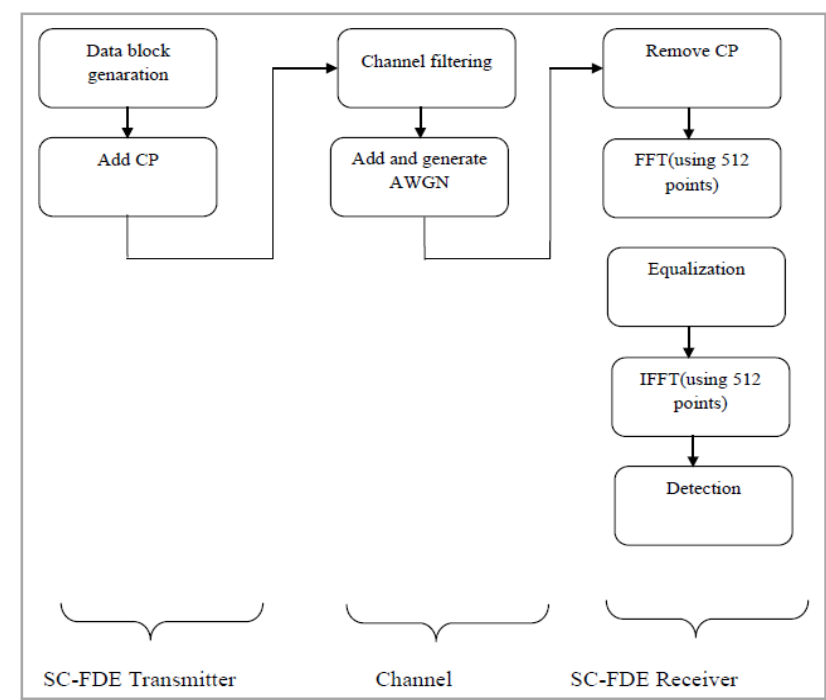

Fig. 3. Block Diagram of SC-FDE Link Level Simulator

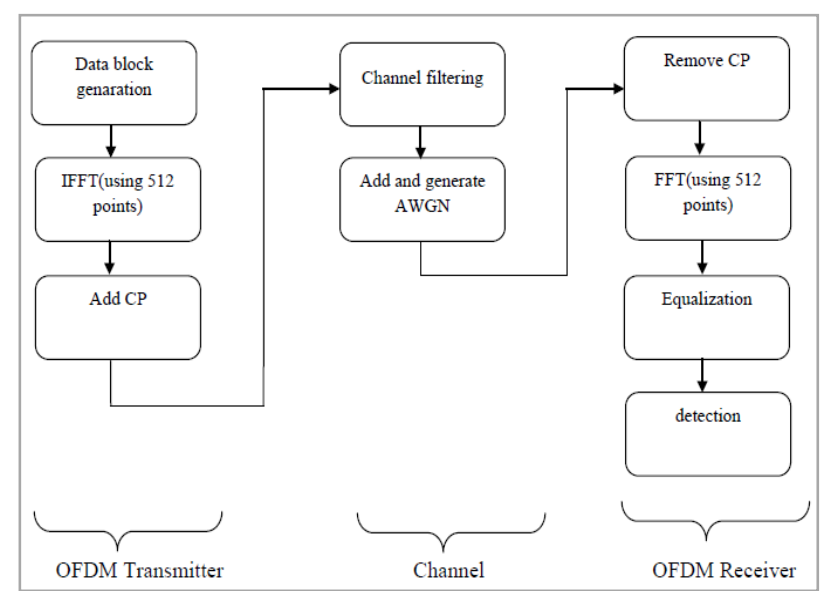

Fig. 4. Block Diagram of OFDM Link Level Simulator

Simulation results show SER for the performance measurement of SC-FDE and OFDM in different scenarios

\subsection{SER for SC-FDE and OFDM using MMSE equalization}

SER measurement of SC-FDE and OFDM were conducted by using three types of channels Pedestrian A, Vehicular A and AWGN. The equalization scheme used for obtaining SER curves is MMSE (Minimum Mean Square Error) (Hamid, 2009). Simulation results show that in case of AWGN channel SC-FDE and OFDM have similar SER performance. However, in case of Pedestrian A and Vehicular A channel, SC-FDE outperforms OFDM.

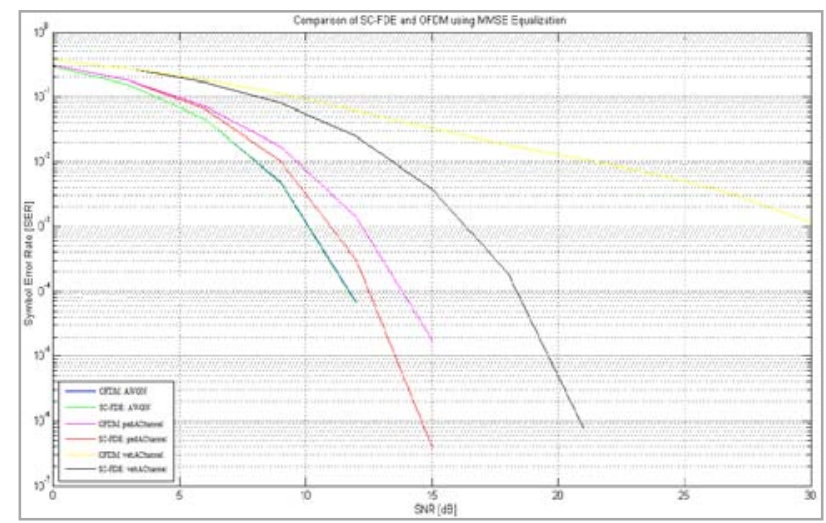

Fig. 5. Comparison of SC-FDE and OFDM using MMSE equalization in Pedestrian A, Vehicular A and AWGN

\subsection{SER for SC-FDE and OFDM using ZF equalization}

In this part was performed calculation of SER using $\mathrm{ZF}$ (Zero Forcing) as equalization scheme for comparison of SCFDE and OFDM in Pedestrian A, Vehicular A and AWGN channel.

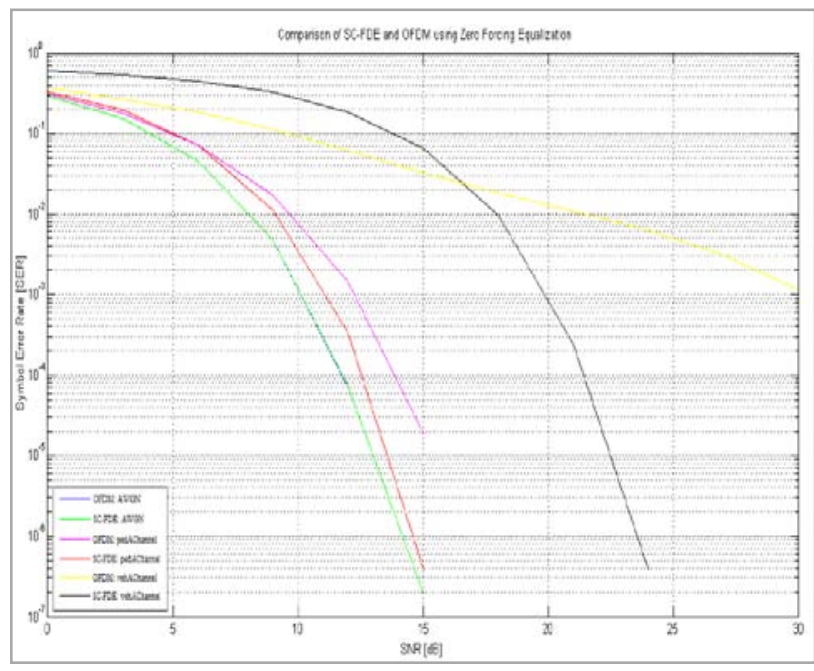

Fig. 6. Comparison of SC-FDE and OFDM using ZF equalization in Pedestrian A, Vehicular A and AWGN

\section{CONCLUSION}

4G networks revolution is still an ongoing process. 3GPP concept will address the IMT-Advanced through a version of LTE called LTE-Advanced, a project which has been researched since 2009 and is expected to end in 2011. LTEAdvanced will be fully compatible with LTE meaning that LTE devices will be able to operate in newer LTE-Advanced networks and vice versa, i.e. the LTE-Advanced devices will operate in older LTE networks. Beyond broadband spectra, LTE-Advanced will increase the performance through more powerful antenna capabilities. For the downlink, the technology will be able to transmit in up to eight levels using $8 \times 8$ configuration for a peak spectra efficiency of 30bps/Hz.

Simulation results show that SC-FDE is superior to OFDM in case of Pedestrian A, Vehicular A channel. From the simulation can be noted that in case of Vehicular A channel usage, OFDM has continuous SER reduction which is significant SER reduction to certain SNR values compared to SC-FDE. Nonetheless, SC-FDE has better performances regarding OFDM as value of SNR is increased.

The drawn conclusion from both comparisons is that compared to ZF MMSE gives better performances.

\section{REFERENCES}

Larmo, A.; Lindström, M.; Meyer, M.; Pelletier, G.; Torsner, J. \& Wiemann, H. (2009). The LTE Link-Layer Design

Dahlman, E.; Parkvall, S.; Skold, J.; \& Beming, P. (2007). $3 G$ Evolution: HSPA and LTE for Mobile Broadband

Fritze, G. (2008). SAE-The Core Network of LTE

Tanvir, M. (2007). Design and analysis for the $3 G$ IP multimedia subsystem

Bahtovski, A. (2011). Master Thesis: Performance Analisis of Next Generation IP Based networks

Sesia, S.; Toufik, I.; Baker, M. (2011). LTE - The UMTS Long Term Evolution: From Theory to Practice

Hamid, S.; Iqbal, M.; Hussain, T. (2009). Comparison between WIMAX and 3GPP LTE 